$30.6 \%$ of Traveller births occurred in the 3 major Dublin maternity hospitals compared to the $35 \%$ of total national births in 2008.

Conclusions In a difficult-to-reach nomadic minority group this study has overcome significant challenges in recruitment. Data to date indicates a downward trend in birth rate in this group.

\section{BIRTH CHARACTERISTICS AND EARLY-LIFE SOCIAL CHARACTERISTICS PREDICT UNEQUAL EDUCATIONAL OUTCOMES: CONSISTENCY ACROSS SWEDISH COHORTS BORN 1915-1929 AND 1973-1980}

doi:10.1136/jech.2010.120956.27

${ }^{1} \mathrm{~A}$ Goodman, ${ }^{2} \mathrm{M} D$ Gisselmann, ${ }^{2} \mathrm{~K}$ Koupil. ${ }^{1}$ Department of Epidemiology and Population Health, London School of Hygiene and Tropical Medicine; ${ }^{2}$ Centre for Health Equity Studies, Stockholm University/Karolinska Institutet, Stockholm, Sweden

Objective To investigate early-life biological and social predictors of educational outcomes, and compare the nature and magnitude of these effects across twentieth century Sweden.

Design Multi-generational data from a representative, populationbased birth cohort, with linkage to routinely collected data.

Setting Sweden.

Participants 9829 Swedish male and females born 1915-1929 and 9465 of their grandchildren born 1973-1980, restricting participants to those who remained alive and in Sweden until age 20.

Characteristics measured at birth Sex, birthweight for gestational age, preterm birth, birth multiplicity, birth order, mother's age, mother's marital status and family social class.

Educational outcomes School achievement was measured using standardised schoolmarks in elementary school. Education continuation was measured as a) senior school attendance and b) entrance to higher education.

Results The predictors of both school achievement and education continuation were very similar in the two cohorts, and effect sizes were usually at least as large in the younger cohort. In both cohorts, the independent predictors of better schoolmarks were: female gender (adjusted effect size 0.35 standard deviations (SD) in 1915-1929, 0.41SD in 1979-1980); higher birthweight for gestational age (0.09SD in 1915-1929, 0.23SD in 1979-1980 for highest vs lowest quintile); lower birth order (eg, 0.33SD in 1915-1925, $0.65 \mathrm{SD}$ in 1973-1980 for birth order 1 vs $4-5$ ); older mother (eg, $0.12 S D$ in $1915-1929,0.34 S D$ in $1973-1980$ for 35-39 years vs $20-24$ years); married mother $(0.14 \mathrm{SD}$ in $1915-1929,0.15 \mathrm{SD}$ in 1973-1980 for married vs unmarried); and higher family social class (eg, 0.39SD in 1915-1929, 0.66SD in 1973-1980 for high/mediate non-manual vs semi/unskilled manual). There were no independent effects of preterm or twin status. The same characteristics predicted education continuation, except that for this outcome the older cohort now showed a marked male advantage and no birthweight effect. Even after adjusting for school achievement, education continuation was still predicted by lower birth order, older mother, married mother and higher social class.

Conclusions Multiple early-life characteristics predicted educational outcomes across the lifecourse. These included size at birth (foetal growth rate) and family composition effects which typically receive far less attention than socio-economic influences. A range of pathways including impaired cognitive development, are likely to mediate these effects. Most effects were remarkably stable across the half-century separating our cohorts, indicating their potential relevance for understanding educational inequalities in populations around the world. Greater understanding of educational inequalities would, in turn, shed light onto a major mechanism whereby health inequalities are created and recreated across generations.

\section{BIRTH SIZE DIFFERENCES BETWEEN WHITE AND PAKISTANI-ORIGIN INFANTS BY GENERATION: RESULTS FROM THE BORN IN BRADFORD COHORT STUDY}

doi:10.1136/jech.2010.120956.28

${ }^{1} \mathrm{~J}$ West, ${ }^{2} \mathrm{D}$ A Lawlor, 'L Fairley, ${ }^{1} \mathrm{~J}$ Wright, ${ }^{3}$ for the Born in Bradford Research Group. ${ }^{1}$ Bradford Institute for Health Research, Bradford Hospitals NHS Trust, Bradford, UK; ${ }^{2}$ MRC Centre for Causal Analyses in Translational Epidemiology, University of Bristol, Oakfield House, Oakfield Grove, Bristol, UK; ${ }^{3}$ www.borninbradford.nhs.uk

Background Previous studies have shown marked differences in birthweight between babies born in the UK of South Asian origin and those of UK origin. Whether such differences persist across generations in contemporary populations, the mechanisms underlying them and the extent to which other dimensions of birth size vary between these two groups is unclear.

Objective To describe differences in term birthweight, head, arm and abdominal circumference and skinfolds between Pakistani origin and white British origin infants and to investigate whether the magnitude of any differences reduces depending on whether the parents and grandparents of Pakistani infants are born in the UK or Pakistan. Design Birth cohort study (Born in Bradford (BiB)).

Setting Bradford, UK.

Participants 1838 white British and 2222 Pakistani mothers recruited to $\mathrm{BiB}$ who completed a questionnaire at 26 weeks gestation and their babies born between Sept 2007 and Nov 2009.

Main outcome measures Birthweight, head, arm and abdominal circumference and skinfolds.

Results Pakistani babies were lighter (mean difference 227.6g, 95\% CI 198.3 to 256.8), had smaller head, arm and abdominal circumferences (mean differences $0.43 \mathrm{~cm}, 95 \%$ CI 0.30 to $0.56 ; 0.22 \mathrm{~cm}, 95 \%$ CI 0.10 to $0.34 ; 1.25 \mathrm{~cm}, 95 \%$ CI 1.02 to 1.39 respectively) and smaller subscapular and triceps skinfold thickness (mean differences $0.22 \mathrm{~mm}, 95 \%$ CI 0.12 to 0.32 and $0.21 \mathrm{~mm}, 95 \%$ CI 0.13 to 0.29 ) than white British infants. Differences remained significant following adjustment for deprivation. Mean birthweight was highest in Pakistani infants when both parents were born in Pakistan (3206 g) and was lowest when both parents were UK-born (3165g). Conclusions These results reaffirm that significant differences in birth size exist between white British and Pakistani origin infants in the UK. Despite the assumption that differences will reduce over successive generations, mean birthweight has not increased in infants of UK-born Pakistani origin parents compared with infants of Pakistani born parents. This suggests that differences may be genetically determined or are affected by epigenetic or persisting behaviour characteristics. Further analysis will include adjustment for additional socio-economic variables, other maternal and family characteristics and birthplace of maternal and paternal grandparents.

\section{DOES A HEAVY BABY BECOME A STRONG CHILD? GRIP STRENGTH AT 4 YEARS IN RELATION TO BIRTHWEIGHT}

\section{doi:10.1136/jech.2010.120956.29}

R Dodds, C Macdonald-Wallis, T Kapasi, A Aihie-Sayer, S Robinson, K Godfrey, C Cooper, N Harvey, H Inskip, the SWS Study Team. MRC Epidemiology Resource Centre, Southampton, UK

Objective Consistent positive relationships have been found between birthweight and grip strength in young, middle-aged and older adults, suggesting that early influences on the growth and development of muscle are important for muscle function later in the lifecourse. However there are limited data in children. We aimed to assess the relationship between birthweight and grip strength in children aged 4 years. 
Design Prospective general population birth cohort study with data from birth to four years, drawn from the Southampton Women's Survey. Grip strength was measured using a Jamar handgrip dynamometer at age four years. We also measured height and weight and assessed body composition (lean mass) using dual energy x-ray absorptiometry. Multiple regression was used to relate grip strength to birthweight allowing for adjustment for confounding factors.

Setting Southampton, UK.

Participants 968 children took part in a sub-study assessing body composition and had their grip strength measured at age four years. Their socio-demographic characteristics were similar to the remainder of the cohort.

Main outcome measure Grip strength in kilograms.

Results Birthweight was positively associated with grip strength, with each kilogram of birthweight being associated with a $0.5 \mathrm{~kg}$ increase in grip strength (95\% CI 0.30 to 0.70). Grip strength was also strongly related to current body size and adjustment of the birthweight relationship for height and weight attenuated the relationship such that it became non-significant with a $1 \mathrm{~kg}$ increase in birthweight being associated with $0.15 \mathrm{~kg}$ ( $95 \%$ CI -0.05 to $0.34 \mathrm{~kg}$ ) increase in grip strength. Adjustment for absolute and percentage lean mass instead of height and weight attenuated the relationship still further such that only a $0.07 \mathrm{~kg}$ ( $95 \% \mathrm{CI}-0.16$ to 0.30 ) increase in grip strength was seen in relation to a $1 \mathrm{~kg}$ increase in birthweight.

Conclusion Birthweight is associated with muscle strength in children aged 4 years. Adjustment for current body size or lean mass explains the relationship suggesting that muscle size is on the causal pathway. Early influences on muscle growth and development appear to impact on grip strength in children as well as adults.

\section{THE EFFECT OF MISSING DATA ON THE RELATIONSHIP BETWEEN LIFECOURSE SOCIO-ECONOMIC POSITION AND VERBAL COGNITIVE ABILITY AT OLDER AGES}

doi:10.1136/jech.2010.120956.30

${ }^{1} \mathrm{R}$ Landy, ${ }^{1} \mathrm{R}$ Hardy, ${ }^{2} \mathrm{~J}$ Head, ${ }^{1} \mathrm{M}$ Richards. ${ }^{1} \mathrm{MRC}$ Unit for Lifelong Health and Ageing, University College London, UK; ${ }^{2}$ Department of Epidemiology and Public Health, University College London, UK

Objective To compare the effects of accounting for different missing data mechanisms in an investigation of the role of lifecourse socioeconomic position (SEP) on later-life verbal cognitive ability.

Design Two UK prospective cohort studies.

Participants A nationally representative sample born in 1946 (NSHD) (original $\mathrm{N}=5362$ ), and a sample of British civil servants (Whitehall II) (original $\mathrm{N}=10308$ ).

Methods Linear regression models were used to test associations between SEP at different life stages and verbal ability. Results from complete case analysis (assuming missing completely at random) were compared with those using multiple imputation (assuming missing at random) and a Heckman selection model (assuming missing not at random) for each cohort.

Main outcome measure Verbal cognitive ability in adulthood; the National Adult Reading Test at age 53 years (NSHD), and the Mill Hill Test at ages 55-79 years (Whitehall II).

Results NSHD: Educational qualifications and head of household occupational SEP at age 53 were significantly related to verbal ability using all missing data methods, after adjusting for sex and cognitive function at age 8 . The effect of childhood SEP was not significant at the $5 \%$ level when using Heckman selection (regression coefficient 0.51 ( $95 \%$ CI -0.25 to 1.27$)$ ) but was significant for complete case analysis (regression coefficient 0.83 (95\% CI 0.11 to 1.54)). Compared with complete case analysis, the coefficients for SEP were generally higher when multiple imputation was used, but the overall conclusions remained the same. The coefficients using Heckman selection differed from those for the complete case and multiple imputation analyses, with lower coefficients for all SEP variables. Whitehall II: Educational qualifications and current occupational SEP were significantly associated with verbal ability for all missing data methods, after adjusting for age, sex, marital status, employment status (working/retired/long-term sick) and number of times the cognitive tests had been taken. The effect of childhood SEP was not significant. The coefficients were generally higher for multiple imputation than complete case analysis, whereas the Heckman selection coefficients were lower for educational qualifications and adult SEP (regression coefficient (95\% CI) -4.46 $(-5.12$ to -3.78$)$ for Heckman selection vs -5.15 ( -5.75 to -4.55$)$ for complete case).

Conclusion Educational qualifications and adult SEP were significant predictors of verbal ability in middle to older age, but results for childhood SEP were inconclusive. Greater differences exist between the results from different missing data methods in the older Whitehall II sample, which may be due to greater selective dropout, which is better accounted for by Heckman selection.

\section{Socio-economic status and cardiovascular risk 031 SOCIO-ECONOMIC PATTERNING OF THE TRENDS IN CARDIOVASCULAR DISEASE RISK FACTORS IN SCOTLAND: SCOTTISH HEALTH SURVEYS 1995 TO 2008}

doi:10.1136/jech.2010.120956.31

${ }^{1} \mathrm{~J}$ W Hotchkiss, ${ }^{1} \mathrm{C}$ A Davies, ${ }^{1} \mathrm{~L}$ Gray, ${ }^{2} \mathrm{C}$ Bromley, ${ }^{3} \mathrm{~S}$ Capewell, ${ }^{1} \mathrm{~A}$ H Leyland. ${ }^{1} \mathrm{MRC}$ CSO Social and Public Health Sciences Unit, Glasgow, UK; ${ }^{2}$ Scottish Centre for Social Research, Edinburgh, UK; ${ }^{3}$ Division of Public Health, University of Liverpool, Liverpool, UK

Background In Scotland, as in other countries, cardiovascular disease (CVD) mortality has substantially declined over time. However, this decline may be slowing among younger groups and there are still large inequalities in mortality between socio-economic groups.

Objectives To examine secular changes in the prevalence of known CVD risk factors in the Scottish population according to socioeconomic position.

Design Representative data on health and health-related behaviours from the Scottish Health Surveys, collected by stratified and clustered probability sampling

Participants Data are available from four surveys: 1995, 1998, 2003 and 2008 (6190, 6656, 5497 and 4202 respondents respectively aged 25-64 years).

Main outcome measures Prevalence of cigarette smoking, excess alcohol consumption (men $>21$, women $>14$ units/week), hypertension (self-reported), diabetes (self-reported) and obesity (measured $\mathrm{BMI} \geq 30 \mathrm{~kg} / \mathrm{m}^{2}$ ).

Methods Prevalences, adjusted for survey weights, and stratified by gender and by gender/age were determined according to the individual's social class (Registrar General's) and educational level (highest qualification), standardised to the European standard population. Time-trends and trends across socio-economic categories were assessed using linear regression. The slope index of inequality (SII) was calculated for each risk factor in every survey. Results In 1995 the prevalence of smoking among professionals was $14.1 \%$ (95\% CI 9.5 to $18.8 \%$ ) compared to unskilled workers $51.0 \%$ (46.1 to $55.8 \%$ ) with SII of 39.0. By 2008 the SII for smoking had decreased slightly to 35.7 , with decreased prevalence in all social classes, except in the unskilled. Self-reported hypertension increased moderately within most educational levels; in men the corresponding SII changed little between the first and last survey (8.8 and 8.2 respectively) while for women it increased from 4.2 to 12.1 . Diabetes prevalence increased in all educational categories. By 2008, the 